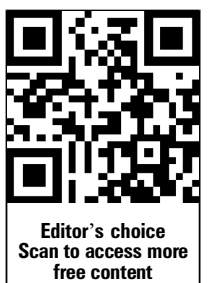

free content
For numbered affiliations see end of article.

\section{Correspondence to} Professor Erik Witvrouw, Aspetar, Qatar Orthopedic and Sports Medicine Hospital, Doha, Qatar;

erik.witvrouw@aspetar.com

Accepted 14 January 2014
To cite: Witvrouw $\mathrm{E}$ Callaghan MJ, Stefanik JJ, et al. Br J Sports Med 2014;48:411-414.

\title{
Patellofemoral pain: consensus statement from the 3rd International Patellofemoral Pain Research Retreat held in Vancouver, September 2013
}

\author{
Erik Witvrouw, ${ }^{1,2}$ Michael J Callaghan, ${ }^{3}$ Joshua J Stefanik, ${ }^{4}$ Brian Noehren, ${ }^{5}$ \\ David M Bazett-Jones, ${ }^{6}$ John D Willson, ${ }^{7}$ Jennifer E Earl-Boehm, ${ }^{8}$ Irene S Davis, ${ }^{9}$ \\ Christopher M Powers, ${ }^{10}$ Jenny McConnell, ${ }^{11}$ Kay M Crossley ${ }^{12}$
}

\section{INTRODUCTION}

Patellofemoral pain (PFP) is often seen in physically active individuals and may account for $25-40 \%$ of all knee problems seen in a sports injury clinic. ${ }^{1}{ }^{2}$ Patellofemoral-related problems occur more frequently in women than in men. ${ }^{3}$ PFP is characterised by diffuse pain over the anterior aspect of the knee and aggravated by activities that increase patellofemoral joint (PFJ) compressive forces, such as squatting, ascending and descending stairs and prolonged sitting, as well as repetitive activities such as running. It, therefore, has a debilitating effect on sufferers' daily lives by reducing their ability to perform sporting and work-related activities pain free. Dye has described PFP as an orthopaedic enigma, and it is one of the most challenging pathologies to manage. ${ }^{4}$ Alarmingly, a high number of individuals with PFP have recurrent or chronic pain. ${ }^{5}$ While physiotherapy interventions for PFP have proven effective compared with sham treatments, treatment results can be disappointing in a proportion of patients. This variability in treatment results may be due to the fact that the underlying factors that contribute to the development of PFP are not being addressed, or are not the same for all patients with PFP. The mission of the 3rd International Patellofemoral Research Retreat was to improve our understanding concerning the factors that contribute to the development and consequently to the treatment of PFP.

The 3rd International Patellofemoral Research Retreat was held in Vancouver, Canada, in September 2013, for 3 days: from 18 September to 21 September. After peer-review for scientific merit and relevance to the retreat, 58 abstracts were accepted for the retreat ( 39 podiums, 8 posters and 11 thematic posters). The podium and poster presentations were grouped into three categories: (1) natural history of PFP and local factors that influence PFP, (2) trunk and distal factors that influence PFP and (3) innovations in rehabilitation of PFP. Presentations in the Natural history and local factors category focused on the prevalence of PFP in different age groups, the relationship between PFP and patellofemoral osteoarthritis (PFOA) and the contribution of surrounding tissues to PFP. Presentations in the Trunk and distal factors category focused on understanding how different factors from different regions (trunk, pelvis, hip and foot and ankle) may contribute to PFJ dysfunction. Presentations in the Innovation in rehabilitation category were dedicated to evaluating outcomes of new treatment options for PFP.

Two keynote speakers were chosen for their scientific contribution in the area of physiotherapy in general, and PFP specifically. Professor Irene Davis from the Harvard Medical School, USA spoke on Alignment and loading: global indicators for patellofemoral pain'. Professor Paul Hodges from the University of Queensland, Australia, addressed the question 'Pain and motor control: what have we learnt?'

\section{Consensus development process}

As with our past two International Patellofemoral Research Retreats, we developed a consensus statement addressing each of the three presentation categories outlined above. This paper is a revision and update of the previous consensus statements published after the first and second international research retreat on PFP in Baltimore 2009, USA. ${ }^{6}$ and Ghent, Belgium in 2011. ${ }^{7}$ Six category consensus leaders facilitated the development of consensus for three specific areas: (1) natural history of PFP and local (knee region) factors that influence PFP (MJC and JJS), (2) trunk and distal factors that influence PFP (BN and DB-J) and (3) innovations in rehabilitation for PFP (JDW and JEE-B).

On the third day of the retreat, participants elected to join one of the three groups, where they discussed and summarised the evidence in their category. Each group was asked to consider two questions: 'What have we learned?' and 'What are the future advances required to understand PFP and its treatment'. Consensus statements were presented for discussion with the entire group, and then refined by the authors of the paper with input from participants.

We present here the 3rd Patellofemoral Pain Consensus Statement. These statements represent the contemporary status of knowledge in the field of PFP, and hence will change over time. This document is developed by researchers for researchers, to stimulate novel studies that will evolve our knowledge of PFP. But in addition to that, we aim to provide clinicians with updated knowledge of PFP, thus enabling them to integrate this knowledge into their clinical practice.

\section{SECTION 1: NATURAL HISTORY OF PFP AND LOCAL (KNEE REGION) FACTORS THAT INFLUENCE PFP}

What have we learned?

Natural history

1. PFP is common in young adolescents, with a high point prevalence of PFP in adolescents between 12 and 17 years of age. ${ }^{8}$ There are no 
data regarding the prevalence or incidence in other populations, except in military personnel ${ }^{1}$ where the annual incidence in men is $3.8 \%$ and in women is $6.5 \%$, with a prevalence of $12 \%$ in men and $15 \%$ in women. ${ }^{1}$

2. The sporting and general populations' true incidence is unknown, and the much cited figure of $25 \%$ is based on figures from sports clinics which have ascertainment bias. Thus, there are insufficient data to confirm the incidence of PFP in these populations. Despite the common assumption that PFP is more common in women, there are a few studies comparing incidence and prevalence between men and women; however, in adolescents, the prevalence is higher in women. ${ }^{9}$ Understanding the different incidence and prevalence rates of PFP between men and women will improve the design of case-control studies.

The PFP/PFOA continuum

3. A subject of major interest is the possibility of a continuum from PFP to PFOA and if there are subgroups/phenotypes of the population who may develop radiographic PFOA or pain, or neither or both. Although it is possible that there is a phenotype of PFP that goes on to develop PFOA, there is no evidence to support this view. There have been no new studies on this subject since the review by Thomas et al, ${ }^{10}$ which was only able to include retrospective evidence in the review.

\section{Patellofemoral osteoarthritis}

4. PFOA is now regarded as a subgroup of knee OA. PFOA prevalence has been described using radiographic evidence in studies of people with knee pain or in community settings $^{11} 12$ or MRI definitions in large epidemiology groups such as the Multicenter Osteoarthritis Study (MOST) and Framingham Osteoarthritis study (FOA) cohorts. $^{13} 14$ Regardless of the methods used to define PFOA, the prevalence is high. Notably, there are new data to challenge the traditional views that PFOA predominantly affects the lateral patellar facet. ${ }^{15}$ These authors demonstrated that medial patellofemoral cartilage damage is highly prevalent, and possibly more prevalent than cartilage damage in the lateral patellofemoral compartment. This finding was consistent across three large epidemiological studies (MOST, FOA and the Boston Osteoarthritis of the Knee Study). Thus, there is a need for research into different mechanisms and risk factors for lateral and medial PFOA.

5. There are new cohorts of people with PFOA on which randomised controlled trials have been performed. ${ }^{16}{ }^{17}$ The interventions used have been bracing and targeted multimodal physiotherapy programmes.

Local (knee region) factors that influence PFP

6. There is new evidence that abnormal structure or alignment of the PFJ may lead to cartilage damage and focal areas of loading and stress manifested as bone marrow lesions (BMLs). Stefanik et $a l^{13}$ reported that knees in the MOST cohort with patella alta and abnormal trochlea morphology were associated with cartilage damage and BML, with similar findings reported from the Osteoarthritis Initiative cohort. ${ }^{18}$

7. The relationship between structure and biomechanics is not known. It is possible that structural abnormalities coupled with poor biomechanics will increase the likelihood of PFP. On the other hand if there is normal structure then the biomechanics may not matter. As yet, no study has examined PFJ structure and mechanics in the same cohort.

8. A systematic review of prospective studies indicates the $\mathrm{Q}$ angle is not a risk factor for $\mathrm{PFP}^{19}$ casting further doubt on the $\mathrm{Q}$ angle's relevance in PFP.
9. There is limited evidence that a variety of local structures contribute to nociception (and potentially pain) in PFP. These include the infrapatellar fat pad in PFP, ${ }^{20}$ increased water content in subchondral patellar bone in athletes ${ }^{21}$ and BMLs in PFOA. ${ }^{14} 22$ There is no new evidence that the retinaculum, which was previously a commonly cited soft tissue problem, contributes to nociception.

\section{Future advances required to understand PFP} and its treatment

1. Large, long-term prospective cohort studies are needed to identify whether there are phenotypes of PFP that goes on to develop PFOA.

2. Future studies should also evaluate the importance of psycho-social factors and central sensitisation in PFP.

3. Identification of different subgroups of people with PFP remains a goal. These subgroups could be based on pain types (eg, nociceptive pain or central sensitisation), or on different structural or biomechanical features. Investigations could then investigate whether different subgroups influence the large individual variation in outcome results after a physiotherapy programme.

4. Inter-relationships between these different psychological, structural and neuromechanical features will impact patient's presentations and treatment responses

\section{SECTION 2: TRUNK AND DISTAL FACTORS THAT INFLUENCE PFP \\ What have we learned?}

Since the publication of the 2011 consensus statement ${ }^{7}$ there have been numerous advances in our understanding of how trunk and lower extremity factors relate to the development of PFP. These advances related particularly to the contribution of the proximal segments (trunk and hip mechanics) to PFP; the increase in new knowledge has been slower in relation to the knee and foot.

\section{Trunk and hip mechanics and PFP}

1. New research adds to the growing consensus that proximal mechanics are altered in women with PFP. This often is observed as excessive hip adduction and/or internal rotation. ${ }^{23-27}$ These altered mechanics have not been reported as consistently in men. Thus, rehabilitation goals might need to be sex-specific. ${ }^{26-28}$

2. A prospective study provided the first evidence that greater hip adduction is present among women who go on to develop PFP. ${ }^{29}$

3. Emerging evidence suggests that trunk mechanics differ between individuals with PFP and those without it. ${ }^{26} 3031$ There are conflicting results on whether contralateral pelvic drop is also greater in patients with PFP compared with pain-free individuals. ${ }^{26} 3031$ Sagittal plane mechanics at the trunk appear to be important in PFP as well. Differences between study results could be partly due to a variety of different kinematic strategies employed by participants, or to differences in the demands of the tasks.

4. The effect of fatigue or exertion on kinematics remains unclear. A recent study found alterations in the sagittal plane, but not in the frontal and transverse planes of the hip and knee following an exhaustive run, ${ }^{30}$ while a different study reported no changes in hip kinematics over the course of a run in those with PFP, despite adaptations observed in a the control group. ${ }^{25}$ This complex area of research is compounded by the onset or progression of pain. 
5. A recent investigation extended what was known about alterations in the frontal and transverse plane mechanics at the hip to the sagittal plane. In this study, hip extension moments were reduced in patients with PFP during running. ${ }^{30}$ In addition, isometric hip extension was weaker in patients with PFP, and this weakness was further exacerbated by an exhaustive run. ${ }^{30}$

6. Further evidence confirms that gluteus medius activation is delayed and of shorter duration in patients with PFP. $^{32} 33$ However, as with most measures of neuromuscular function, not all studies report consistent findings, ${ }^{34}$ most likely due to differences in methodology and patient populations.

\section{Knee and foot mechanics and PFP}

7. Functional MRI, used to map quadriceps activation, questions the long-held belief that altered quadriceps activation patterns are evident in patients with PFP. $^{35}$ The implication of this finding is yet to be elucidated.

8. Lower extremity kinetics studies have demonstrated reduced peak knee extension moments in patients with PFP during walking but not during running. ${ }^{30}$ 36-38

9. The importance of rearfoot eversion remains unclear. Patients with PFP use more of their available rearfoot eversion during gait than do healthy control participants ${ }^{39}$ and greater rearfoot eversion may be related to hip adduction in individuals with PFP. $^{40}$ However, to date, no prospective studies have identified rearfoot eversion as a predictor of PFP. $^{29}$

10. Greater tibial internal rotation, but not foot mechanics measured with a multisegment foot model, was observed in people with PFP group compared with controls. ${ }^{31}$ Alterations in tibial rotation may provide a potent ial link between PFP and distal factors. ${ }^{31}$

\section{Future advances required to understand PFP and its treatment}

1. Similar to the recommendations for local factors, identification of subgroups remains the 'holy grail' for PFP research. These studies could yield insights into the pathophysiology of PFP and provide targets for treatment (ie, addressing strength or gait impairments). However, such studies likely require larger numbers of patients than can be obtained relatively quickly in single centres.

2. The contribution of the gluteal muscles and quadriceps to PFP has been well studied in women. However, the influence of other muscles (their strength, activation, endurance) such as the hamstring, hip adductor and distal muscles such as the posterior tibialis and intrinsic foot muscles need to be investigated. ${ }^{41}$ Studies are also needed in men (with and without PFP) to characterise the role of all muscles.

3. A more comprehensive approach to muscle activation should be considered in the design of future studies. Specifically, studies should examine the coordination of muscle activation patterns across a number of joints, rather than just across a single joint.

4. Investigations are needed to assess the inter-relationships between different elements of muscle functions (eg, muscle activation patterns, eccentric strength, rate of force development and muscle endurance) with joint mechanics.

5. Important observations on injury mechanics have been gained from examining peak joint angles. Future investigations should consider the contribution of other variables (eg, joint excursions, average joint angles and joint velocities) in patients with PFP. Any abnormal joint kinematics observed in patients with PFP could be targets for treatment.
6. Inconsistencies between studies may be reduced if all investigators used a consistent battery of tasks (ie, walking, running, single-leg squat), which would facilitate more meaningful comparisons and meta-analyses. However, we also need studies to compare mechanics across a variety of tasks within the same cohort, to help define the tasks likely to reveal abnormal mechanics.

7. Clinically relevant measures of kinematics should also be developed and included in biomechanical studies when possible.

8. The effects of gait retraining interventions targeted at the hip, on foot biomechanics should be evaluated. ${ }^{40}$

9. Prospective studies are needed to:

a. Determine the influence of trunk and pelvis mechanics, in addition to lower limb mechanics, on the development of PFP in the men and women.

b. Assess hip strength and activation as a contributor to PFP. The current evidence for the association comes from crosssectional studies. Prospective studies could lend important insights into whether strength, activation or mechanics are altered before an individual develops PFP.

\section{SECTION 3: INNOVATIONS IN REHABILITATION OF PFP What have we learned?}

1. Previous consensus statements ${ }^{7}$ have highlighted that conservative interventions including therapeutic exercises (eg, open and closed kinetic chain exercises), multimodal physiotherapy, foot-orthoses and patellar taping ${ }^{42-52}$ can reduce PFP and increase self-reported function, in the short term ( $<1$ year). However, at 1 year from treatment, a significant proportion $(40 \%)$ of people with PFP did not feel that exercise interventions moderately improved symptoms or led to full recovery from PFP. ${ }^{43} 44$

2. Since 2011 (the last research retreat) a randomised clinical trial has identified that a conservative intervention, including therapeutic exercises, may prevent the development of PFP in an active population. ${ }^{53}$

3. Since 2011, a number of studies have investigated more novel interventions, including hip muscle retraining and movement retraining.

4. Therapeutic exercises focused on strengthening posterolateral hip muscles reduce pain and improve function when performed alone, or in combination with multimodal therapy. These studies examined only short-term outcome with a maximum of 1 year follow-up. ${ }^{54-58}$

5. Movement feedback interventions during treadmill running may change movement patterns of people with PFP during weight-bearing activities and these kinematic adjustments may reduce PFP symptoms. ${ }^{59-63}$ However, increasing hip abductor and knee extensor muscle strength does not affect altered lower extremity kinematics associated with PFP. ${ }^{56} 6364$

6. Use of physical agents (therapeutic modalities) has not shown benefit in patients with PFP compared with controls. $^{65}$

\section{Future advances required to understand PFP and its treatment}

1. Interventions should be tailored to specific populations of patients (ie, adolescents, athletes, military, older adults, etc) as the pathology/aetiology in these groups may differ.

2. The methods for participant recruitment should be well described to include the site (ie, school, clinic, sports) and method of recruitment (ie, response to advertisement, referral from healthcare provider, chart review). 
3. Poor long-term outcomes could be due to patients discontinuing their exercise programmes once they are discharged from formal rehabilitation. Future trials should monitor compliance/adherence carefully and investigate exercise programmes that last $>1$ year.

4. While multimodal interventions are frequently successful, a simplified intervention that can be part of a patient's regular routine and maintained for a longer period of time should be investigated. This may improve long-term adherence and reduce chronicity.

5. Further research could determine whether interventions can change movement patterns thought to contribute to the aetiology or chronicity of PFP symptoms and can reduce the burden of PFP in the long term.

6. The mechanism for the benefit of many conservative treatments, including additional posterolateral hip strengthening, is unknown.

7. The short-term and long-term effectiveness of interventions for PFP among adolescents and older populations is an area in need of research.

8. Investigators should use a standard set of outcome measures and the timing of measurement should be consistent across trials to facilitate future comparisons. Self-reported pain (VAS), function, recovery (global rating of change) and quality-of-life should be included.

9. As pain is one of the primary outcome measures, it should be evaluated in multiple dimensions (ie, physical, psychological, quality-of-life, kinesiophobia, catastrophising, mood, etc).

10. There have been initial attempts to classify patients by aetiological subgroup. Additional work should continue in this area to attempt to classify patients for targeted interventions.

\section{SECTION 4: GENERAL RECOMMENDATIONS FOR FUTURE DIRECTIONS}

Researchers should consider more clearly detail how many individuals they had to exclude to obtain the patients in their study. These data would help establish how common a particular injury pattern or subgroup is and would also provide a guide for generalisability of the study.

In addition, those studies investigating biomechanical factors should strive to clarify their results in terms of statistical and clinical significance. This not only helps further research to focus interventions likely to have a beneficial outcome but will help clinicians to interpret the likely outcome of an intervention.

More generally speaking, the International Patellofemoral Pain Research Conference supports the 'EQUATOR' guidelines (http://www.equator-network.org) for reporting and encourages investigators to follow the 'PRISMA', 'STROBE' and 'CONSORT' recommendations.

\footnotetext{
Author affiliations

${ }^{1}$ Aspetar, Qatar Orthopedic and Sports Medicine Hospital, Doha, Qatar

${ }^{2}$ Department of Rehabilitation Sciences and Physiotherapy, Ghent University, Ghent, Belgium

${ }^{3}$ Manchester Academic Health Science Centre (MAHSC), University of Manchester, Manchester, UK

${ }^{4}$ College of Health and Rehabilitation Sciences, Boston University School of Medicine, Boston, Massachusetts, USA

${ }^{5}$ Division of Physical Therapy, University of Kentucky, Lexington, Kentucky, USA

${ }^{6}$ Department of Physical Therapy, Carroll University, Waukesha, Wisconsin, USA

${ }^{7}$ Department of Physical Therapy, East Carolina University, Greenville,

North Carolina, USA

${ }^{8}$ Department of Kinesiology, University of Wisconsin-Milwaukee, Milwaukee, Wisconsin, USA

${ }^{9}$ Department of Physical Medicine and Rehabilitation, Harvard Medical School, Cambridge, Massachusetts, USA
}

${ }^{10}$ Division of Biokinesiology and Physical Therapy, University of Southern California, Los Angeles, California, USA

${ }^{11}$ McConnell and Clements Physiotherapy, Mosman, Australia

${ }^{12}$ Division of Physiotherapy, School of Health \& Rehabilitation Sciences, University of Queensland, Brisbance, Queensland, Australia

\section{Competing interests None.}

Provenance and peer review Commissioned; internally peer reviewed.

\section{REFERENCES}

1 Boling M, Padua D, Marshall S, et al. Gender differences in the incidence and prevalence of patellofemoral pain syndrome. Scand J Med Science Sports 2010;20:725-30.

2 Kannus $\mathrm{P}$, Aho $\mathrm{H}$, Jarvinen $\mathrm{M}$, et al. Computerized recording of visits to an outpatient sports clinic. Am J Sports Med 1987;15:79-85.

3 Taunton JE, Ryan MB, Clement DB, et al. A retrospective case-control analysis of 2002 running injuries. Br J Sports Med 2002;36:95-101.

4 Dye S. Patellofemoral pain current concepts: an overview. Sports Med Arthrosc Rev 2001;9:264-72.

5 Stathopulu E, Baildam E. Anterior knee pain: a long-term follow up. Rheumatology (Oxford) 2003:42:380-2

6 Davis IS, Powers CM. Patellofemoral pain syndrome: proximal, distal, and local factors an International Research Retreat. JOSPT 2010;40:a1-a48.

7 Powers CM, Bolgla LA, Callaghan MJ, et al. Patellofemoral pain; proximal, distal, and local factors. 2nd International Research Retreat. JOSPT 2012;42:a1-a20.

8 Rathleff MS, Skuldbøl SK, Rasch MN, et al. Care-seeking behaviour of adolescents with knee pain: a population-based study among 504 adolescents. BMC Musculoskelet Disord 2013;14:225

9 Mølgaard C, Rathleff MS, Simonsen O. Patellofemoral pain syndrome and its association with hip, ankle, and foot function in 16- to 18-year-old high school students: a single-blind case-control study. J Am Podiatr Med Assoc 2011;101:215-22.

10 Thomas MJ, Wood L, Selfe J, et al. Anterior knee pain in younger adults as a precursor to subsequent patellofemoral osteoarthritis: a systematic review. BMC Musculoskelet Disord 2010;11:201.

11 Duncan $\mathrm{R}$, Peat $\mathrm{G}$, Thomas $\mathrm{E}$, et al. Does isolated patellofemoral osteoarthritis matter? Osteoarthritis Cartilage 2009;17:1151-5.

12 McAlindon TE, Snow S, Cooper C, et al. Radiographic patterns of osteoarthritis of the knee joint in the community: the importance of the patellofemoral joint. Ann Rheum Dis 1992:51:844-9.

13 Stefanik JJ, Zhu Y, Zumwalt AC, et al. Association between patella alta and the prevalence and worsening of structural features of patellofemoral joint osteoarthritis: the multicenter osteoarthritis study. Arthritis Care Res 2010;62:1258-65.

14 Stefanik JJ, Niu J, Gross KD, et al. Using magnetic resonance imaging to determine the compartmental prevalence of knee joint structural damage. Osteoarthritis Cartilage 2013;21:695-9.

15 Gross KD, Niu J, Stefanik JJ, et al. Breaking the law of valgus: The surprising and unexplained prevalence of medial patellofemoral cartilage damage. Ann Rheum Dis 2012:71:1827-32.

16 Crossley KM, Vicenzino B, Pandy MG, et al. Targeted physiotherapy for patellofemoral joint osteoarthritis: a protocol for a randomised, single-blind controlled trial. BMC Musculoskelet Disord 2008:9:122.

17 Hunter DJ, Harvey W, Gross KD, et al. A randomized trial of patellofemoral bracing for treatment of patellofemoral osteoarthritis. Osteoarthritis Cartilage 2011;19:792-800

18 Jungmann PM, Tham SC, Liebl H, et al. Association of trochlear dysplasia with degenerative abnormalities in the knee: data from the Osteoarthritis Initiative. Skeletal Radiol 2013;42:1383-92.

19 Lankhorst NE, Bierma-Zeinstra SMA, Van Middelkoop M. Risk factors for patellofemoral pain syndrome: A systematic review. J Orthopaedic Sports Physical Therapy 2012;42:81-A12.

20 Dragoo JL, Johnson C, McConnell J. Evaluation and treatment of disorders of the infrapatellar fat pad. Sports Med 2012:42:51-67.

$21 \mathrm{Ho} \mathrm{KY,} \mathrm{Hu} \mathrm{HH}$, Colletti PM, et al. Running-induced patellofemoral pain fluctuates with changes in patella water content. Eur J Sport Sci 2013.

22 Zhang $Y$, Nevitt M, Niu J, et al. Fluctuation of knee pain and changes in bone marrow lesions, effusions, and synovitis on magnetic resonance imaging. Arthritis Rheum 2011;63:691-9.

23 McKenzie K, Galea V, Wessel J, et al. Lower extremity kinematics of females with patellofemoral pain syndrome while stair stepping. J Orthop Sports Phys Ther 2010:40:625-32

24 Wirtz AD, Willson JD, Kernozek TW, et al. Patellofemoral joint stress during running in females with and without patellofemoral pain. Knee 2012;19:703-8.

25 Noehren B, Sanchez Z, Cunningham T, et al. The effect of pain on hip and knee kinematics during running in females with chronic patellofemoral pain. Gait Posture 2012:36:596-9. 
26 Nakagawa TH, Moriya ET, Maciel CD, et al. Trunk, pelvis, hip, and knee kinematics, hip strength, and gluteal muscle activation during a single-leg squat in males and females with and without patellofemoral pain syndrome. J Orthop Sports Phys Ther 2012:42:491-501.

27 Nakagawa TH, Moriya ET, Maciel CD, et al. Frontal plane biomechanics in males and females with and without patellofemoral pain. Med Sci Sports Exerc 2012:44:1747-55.

28 Willy RW, Manal KT, Witvrouw EE, et al. Are mechanics different between male and female runners with patellofemoral pain? Med Sci Sports Exerc 2012;44:2165-71.

29 Noehren B, Hamill J, Davis I. Prospective evidence for a hip etiology in patellofemoral pain. Med Sci Sports Exerc 2013;45:1120-4.

30 Bazett-Jones DM, Cobb SC, Huddleston WE, et al. Effect of patellofemoral pain on strength and mechanics after an exhaustive run. Med Sci Sports Exerc 2013;45:1331-9.

31 Noehren B, Pohl MB, Sanchez Z, et al. Proximal and distal kinematics in female runners with patellofemoral pain. Clin Biomech 2012;27:366-71.

32 Barton CJ, Lack S, Malliaras $\mathrm{P}$, et al. Gluteal muscle activity and patellofemoral pain syndrome: a systematic review. Br J Sports Med 2013;47:207-14.

33 Aminaka N, Pietrosimone BG, Armstrong CW, et al. Patellofemoral pain syndrome alters neuromuscular control and kinetics during stair ambulation. J Electromyogr Kinesiol 2011;21:645-51.

34 O'Sullivan K, Herbert E, Sainsbury D, et al. No difference in gluteus medius activation in women with mild patellofemoral pain. J Sport Rehabil 2012;21:110-18

35 Pattyn E, Verdonk P, Steyaert A, et al. Muscle functional MRI to evaluate quadriceps dysfunction in patellofemoral pain. Med Sci Sports Exerc 2013:45:1023-9.

36 Paoloni $\mathrm{M}$, Mangone $\mathrm{M}$, Fratocchi $\mathrm{G}$, et al. Kinematic and kinetic features of normal level walking in patellofemoral pain syndrome: more than a sagittal plane alteration. J Biomech 2010;43:1794-8.

37 Salsich GB, Long-Rossi F. Do females with patellofemoral pain have abnormal hip and knee kinematics during gait? Physiother Theory Pract 2010;26:150-9.

38 Claudon B, Poussel M, Billon-Grumillier C, et al. Knee kinetic pattern during gait and anterior knee pain before and after rehabilitation in patients with patellofemoral pain syndrome. Gait Posture 2012;36:139-43.

39 Rodrigues P, TenBroek T, Hamill J. Runners with anterior knee pain use a greater percentage of their available pronation range of motion. J Appl Biomech 2013;29:141-6.

40 Barton $\mathrm{CJ}$, Levinger $\mathrm{P}$, Crossley $\mathrm{KM}$, et al. The relationship between rearfoot, tibial and hip kinematics in individuals with patellofemoral pain syndrome. Clin Biomech 2012;27:702-5.

41 Besier TF, Fredericson $\mathrm{M}$, Gold $\mathrm{GE}$, et al. Knee muscle forces during walking and running in patellofemoral pain patients and pain-free controls. J Biomech 2009:42:898-905.

42 Crossley K, Bennell K, Green S, et al. Physical therapy for patellofemoral pain: a randomized, double-blinded, placebo-controlled trial. Am I Sports Med 2002:30:857-65.

43 Collins N, Crossley K, Beller E, et al. Foot orthoses and physiotherapy in the treatment of patellofemoral pain syndrome: randomised clinical trial. $\mathrm{Br}$ I Sports Med 2009;43:169-71.

44 van Linschoten R, van Middelkoop M, Berger MY, et al. Supervised exercise therapy versus usual care for patellofemoral pain syndrome: an open label randomised controlled trial. BMJ 2009:339:b4074.

45 Syme G, Rowe P, Martin D, et al. Disability in patients with chronic patellofemoral pain syndrome: a randomised controlled trial of VMO selective training versus general quadriceps strengthening. Man Ther 2009:14:252-63.

46 Song CY, Lin YF, Wei TC, et al. Surplus value of hip adduction in leg-press exercise in patients with patellofemoral pain syndrome: a randomized controlled trial. Phys Ther 2009;89:409-18.
47 Bakhtiary AH, Fatemi E. Open versus closed kinetic chain exercises for patellar chondromalacia. Br J Sports Med 2008;42:99-102.

48 Herrington L, Al-Sherhi A. A controlled trial of weight-bearing versus non-weight-bearing exercises for patellofemoral pain. J Orthop Sports Phys Ther 2007:37:155-60.

49 Witvrouw E, Lysens R, Bellemans J, et al. Open versus closed kinetic chain exercises for patellofemoral pain. Am J Sports Med 2000;28:687-94.

50 Witvrouw E, Danneels L, Van Tiggelen D, et al. Open versus closed kinetic chain exercises in patellofemoral pain: a 5-year prospective randomized study. Am I Sports Med 2004:32:1122-30

51 Whittingham M, Palmer S, Macmillan F. Effects of taping on pain and function in patellofemoral pain syndrome: a randomized controlled trial. J Orthop Sports Phys Ther 2004;34:504-10.

52 Clark DI, Downing N, Mitchell J, et al. Physiotherapy for anterior knee pain: a randomised controlled trial. Ann Rheum Dis 2000;59:700-4.

53 Coppack RJ, Etherington J, Wills AK. The effects of exercise for the prevention of overuse anterior knee pain: a randomized controlled trial. Am I Sports Med 2011:39:940-8

54 Fukuda TY, Melo WP, Zaffalon BM, et al. Hip posterolateral musculature strengthening in sedentary women with patellofemoral pain syndrome: a randomized controlled clinical trial with 1-year follow-up. J Orthop Sports Phys Ther 2012;42:823-30.

55 Dolak KL, Silkman C, Medina McKeon J, et al. Hip strengthening prior to functional exercises reduces pain sooner than quadriceps strengthening in females with patellofemoral pain syndrome: a randomized clinical trial. J Orthop Sports Phys Ther 2011:41:560-70

56 Earl JE, Hoch AZ. A proximal strengthening program improves pain, function, and biomechanics in women with patellofemoral pain syndrome. Am I Sports Med 2011;39:154-63.

57 Khayambashi K, Mohammadkhani Z, Ghaznavi K, et al. The effects of isolated hip abductor and external rotator muscle strengthening on pain, health status, and hip strength in females with patellofemoral pain: a randomized controlled trial. J Orthop Sports Phys Ther 2012;42:22-9.

58 Khayambashi K, Fallah A, Movahedi A, et al. Posterolateral hip strengthening verses quadriceps strengthening for patellofemoral pain: a randomized controlled trial [abstract]. Br J Sports Med 2014;6:e1.abstract 28.

59 Cheung RT, Davis IS. Landing pattern modification to improve patellofemoral pain in runners: a case series. J Orthop Sports Phys Ther 2011;41:914-19.

60 Noehren B, Scholz J, Davis I. The effect of real-time gait retraining on hip kinematics, pain and function in subjects with patellofemoral pain syndrome. $\mathrm{Br} J$ Sports Med 2011:45:691-6.

61 Salsich GB, Graci V, Maxam DE. The effects of movement pattern modification on lower extremity kinematics and pain in women with patellofemoral pain. J Orthop Sports Phys Ther 2012;42:1017-24.

62 Willy RW, Scholz JP, Davis IS. Mirror gait retraining for the treatment of patellofemoral pain in female runners. Clin Biomech (Bristol, Avon) 2012:27:1045-51.

63 Willy RW, Davis IS. The effect of a hip-strengthening program on mechanics during running and during a single-leg squat. J Orthop Sports Phys Ther 2011; 41:625-32.

64 Ferber R, Kendall KD, Farr L. Changes in knee biomechanics after a hip-abductor strengthening protocol for runners with patellofemoral pain syndrome. J Athl Train 2011;46:142-9.

65 Lake DA, Wofford NH. Effect of therapeutic modalities on patients with patellofemoral pain syndrome: a systematic review. Sports Health 2011;3:182-9. 